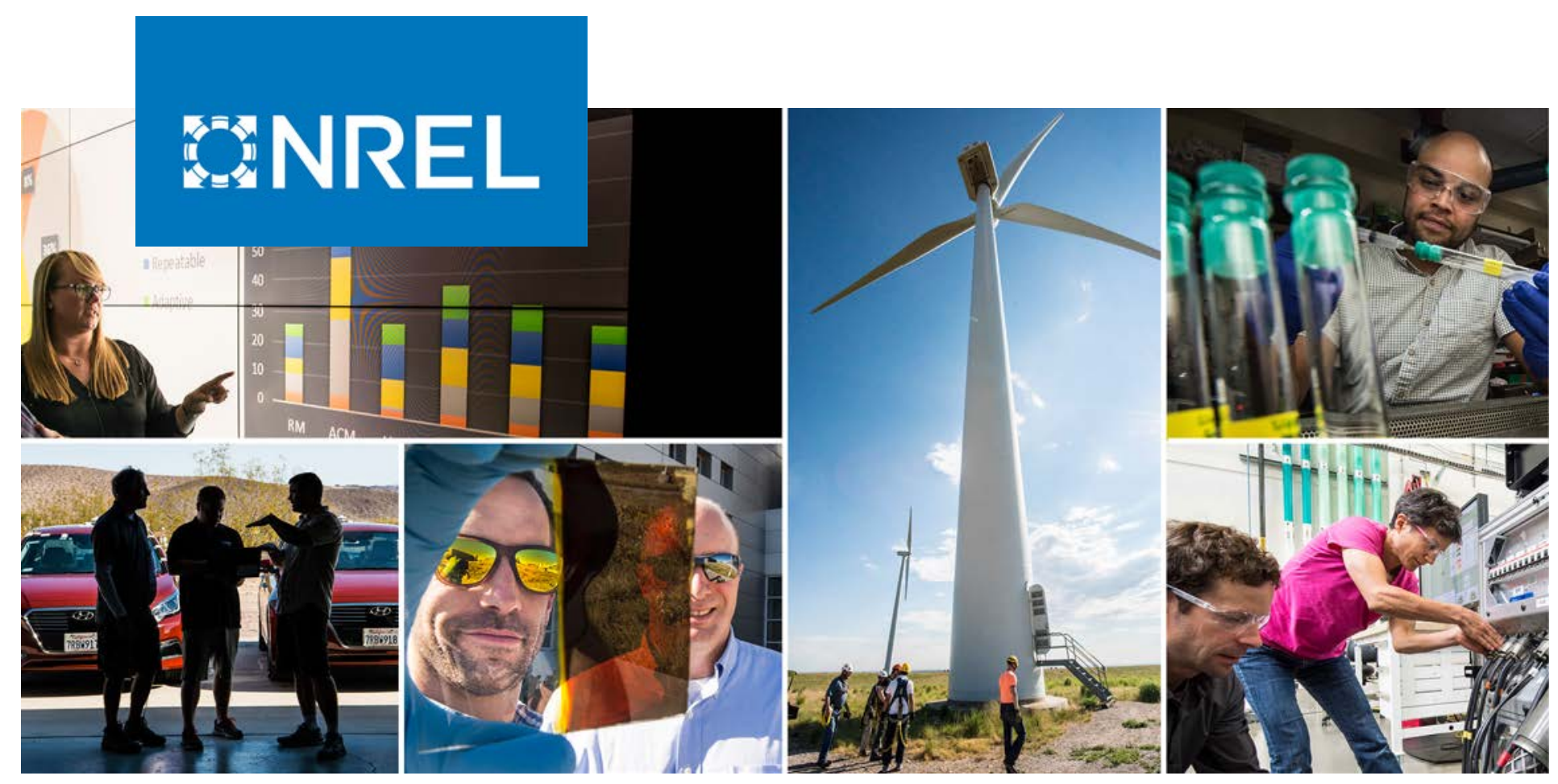

\title{
Real-Time Volume and Turning Movements from Probe Data
}

\section{Cooperative Research and Development Final Report}

CRADA Number: CRD-16-614

NREL Technical Contacts: Stanley Young,

Venu Garikapati, and Yi Hou

NREL is a national laboratory of the U.S. Department of Energy

Office of Energy Efficiency \& Renewable Energy

Operated by the Alliance for Sustainable Energy, LLC

This report is available at no cost from the National Renewable Energy Laboratory (NREL) at www.nrel.gov/publications.
Technical Report

NREL/TP-5400-77458

August 2020 


\section{GNREL}

\section{Real-Time Volume and Turning Movements from Probe Data}

Cooperative Research and Development Final Report

\section{CRADA Number: CRD-16-614}

NREL Technical Contacts: Stanley Young, Venu Garikapati, and Yi Hou

\section{Suggested Citation}

Young, Stanley, Venu Garikapati, and Yi Hou. 2020. Real-Time Volume and Turning Movements from Probe Data: Cooperative Research and Development Final Report, CRADA Number CRD-16-614. Golden, CO: National Renewable Energy Laboratory. NREL/TP-540-77458. https://www.nrel.gov/docs/fy20osti/77458.pdf.

NREL is a national laboratory of the U.S. Department of Energy Office of Energy Efficiency \& Renewable Energy Operated by the Alliance for Sustainable Energy, LLC

This report is available at no cost from the National Renewable Energy Laboratory (NREL) at www.nrel.gov/publications.

Contract No. DE-AC36-08GO28308
Technical Report NREL/TP-5400-77458 August 2020

National Renewable Energy Laboratory 15013 Denver West Parkway Golden, CO 80401 303-275-3000 • www.nrel.gov 


\section{NOTICE}

This work was authored [in part] by the National Renewable Energy Laboratory, operated by Alliance for Sustainable Energy, LLC, for the U.S. Department of Energy (DOE) under Contract No. DE-AC36-08GO28308. Funding provided by U.S. Department of Energy Office of Energy Efficiency and Renewable Energy Vehicle Technologies Office. The views expressed herein do not necessarily represent the views of the DOE or the U.S. Government.

This work was prepared as an account of work sponsored by an agency of the United States Government. Neither the United States Government nor any agency thereof, nor any of their employees, nor any of their contractors, subcontractors or their employees, makes any warranty, express or implied, or assumes any legal liability or responsibility for the accuracy, completeness, or any third party's use or the results of such use of any information, apparatus, product, or process disclosed, or represents that its use would not infringe privately owned rights. Reference herein to any specific commercial product, process, or service by trade name, trademark, manufacturer, or otherwise, does not necessarily constitute or imply its endorsement, recommendation, or favoring by the United States Government or any agency thereof or its contractors or subcontractors. The views and opinions of authors expressed herein do not necessarily state or reflect those of the United States Government or any agency thereof, its contractors or subcontractors.

This report is available at no cost from the National Renewable Energy Laboratory (NREL) at www.nrel.gov/publications.

U.S. Department of Energy (DOE) reports produced after 1991 and a growing number of pre-1991 documents are available free via www.OSTI.gov.

Cover Photos by Dennis Schroeder: (clockwise, left to right) NREL 51934, NREL 45897, NREL 42160, NREL 45891, NREL 48097, NREL 46526.

NREL prints on paper that contains recycled content. 


\section{Cooperative Research and Development Final Report}

Report Date: June 8, 2020

In accordance with requirements set forth in the terms of the CRADA agreement, this document is the final CRADA report, including a list of subject inventions, to be forwarded to the DOE Office of Scientific and Technical Information as part of the commitment to the public to demonstrate results of federally funded research.

Parties to the Agreement: University of Maryland

CRADA Number: CRD-16-614

CRADA Title: Real-time Volume and Turning Movements from Probe Data

Joint Work Statement Funding Table showing DOE commitment:

No National Renewable Energy Laboratory (NREL) Shared Funds.

\begin{tabular}{|c|c|}
\hline Estimated Costs & NREL Shared Resources \\
\hline Year 1 & $\$ 00.00$ \\
\hline TOTALS & $\$ 00.00$ \\
\hline
\end{tabular}

Abstract of CRADA Work:

NREL provided technical assistance for the I-95 Corridor Coalition to develop traffic volume estimates based upon third party probe vehicle data and other sources. The overall objective to accelerate the timeframe for achieving viable estimates was achieved.

\section{Summary of Research Results:}

The purpose of this section is to capture the original or modified scope, completed work and outcomes of this project. DOE requires that this template address all the planned tasks in the Joint Work Statement.

\section{PHASE I}

\section{Task 1 Project Management}

NREL directly assisted UMD CATT in project management. This included assisting with periodic reporting to the I-95 Corridor Coalition about the project status and ensuring overall project delivery. Management activities included ensuring conformance to anticipated timeline, budget, deliverables and milestones, and mitigating any disruptions to such as they arise. This also included participation in presentations and updates to the Coalition from time to time via webinars or conference calls. 


\section{Task 2 Specifications and Requirements}

\section{Task 2-1 Use Survey}

Task 2-1 involved establishing the project framework through a member survey. The survey helped to define project direction as well as the needs and expectations of practitioners with respect to data uses and data fidelity. A primary takeaway from the survey was that volume estimates needed to be within $10 \%$ with respect to capacity to be considered useful for highway operations. ${ }^{1}$

NREL supported Task 2-1 by reviewing survey extent, assisting with the interpretation of the results, and compilation and review of the survey report. NREL assisted in soliciting input from outside the Coalition through its network of transportation energy interests as well as the transportation modeling community. It also participated as a user of volume data, providing input concerning energy analysis and sustainability application concerns.

\section{Task 2-2 Recommended Specifications}

The research team, in consultation with the Coalition and industry partners, created specifications for accuracy, format and delivery of volume and turning movement data. NREL led the formulation of the recommended specifications under the direction of Dr. Young, who was the primary technical architect of the Vehicle Probe Project (VPP) and its validation program prior to joining NREL. Input into this process included feedback from the I95 Coalition (as per the survey referenced above), input from the steering committee which met approximately quarterly to review the research results, and from discussion between and amongst the researchers as they progressed through the project.

The research team conferred upon target metrics with respect to three accuracy measures that are summarized in Figure 1. These metrics were discussed throughout the research review process.

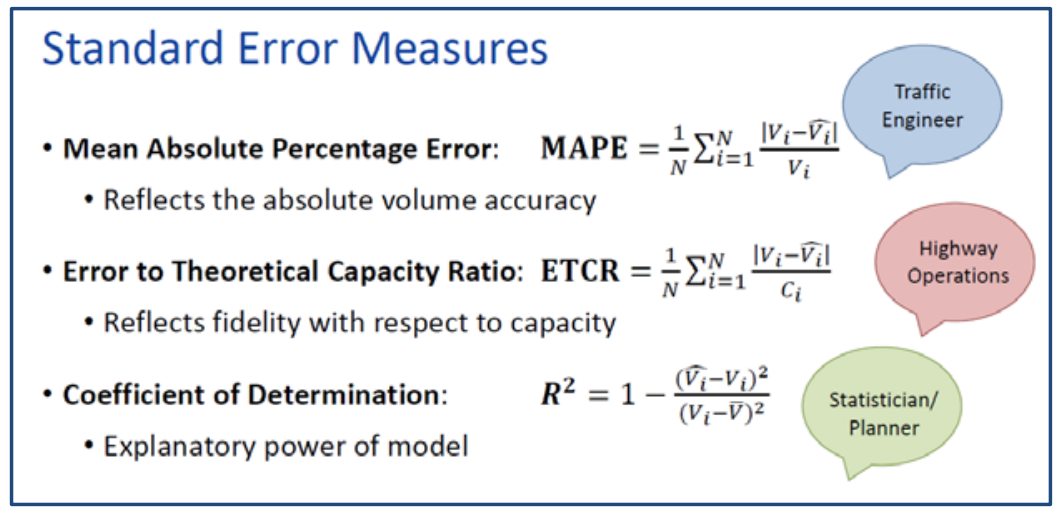

Figure 1 - Standard Error measures used in volume estimation research

Figure 1 summarizes recommended targets for outsourced volume data specifications based on research and analysis from the Colorado, Maryland, Florida, and New Hampshire data sets explored, as well as lessons learned from parallel research conducted by Texas Transportation Institute (TTI) in Minnesota on low volume roads.

\footnotetext{
${ }^{1}$ https://www.nrel.gov/docs/fy18osti/70938.pdf
} 


\section{How good is good enough?}

- Mean absolute percent error (MAPE)

- $10 \%$ to $15 \%$ in high-volume

- $15 \%$ to $25 \%$ in mid-volume

- $25 \%$ to ??\% in low-volume

- Coefficient of Determination, $\mathrm{R}^{2}$

- Good: greater than $70 \%$

- Better: greater than $80 \%$

- Best: greater than $90 \%$

- Error to Capacity (ETCR) or Max Flow (EMFR)

- $\{$ Use if you care ONLY about measuring accurate volumes near capacity\}

- Useful: less than $10 \%$

- Target: less than 5\%

Figure 2 - Accuracy targets for the three primary error metrics (source: Turner, NATMEC 2018)

\section{Task 2-3 Freight (Origin \& Destination) Data and Practices}

Task 2-3 involved exploration of the base data set for freight applications. UMD completed this task, and the information gained was summarized in a published journal article (Sekula et al., 2018). NREL participated by reviewing UMD's work and attending a webinar on this topic.

\section{Task 3 Testbed Initialization}

The efforts outlined in Task 3 of the work statement were predicated on industry partners providing volume estimates, and that UMD and NREL would compare these volume estimates to acknowledged reference data. However, as the project progressed, industry partners instead provided base, unprocessed data from which researchers at UMD and NREL created algorithms and methods to estimate traffic volumes. As a result, the testbed creation and execution took on a different form, but the overall objective was accomplished. The following sections describe the testbed initialization process, with loose adherence to the original task structure.

\section{Task 3-1 Validation Methodology}

The research team cooperatively developed a validation methodology to evaluate the quality and potential of probe data to estimate volume and turning movements. The original SOW anticipated vendors would independently develop volume estimates while UMD and NREL provided the framework and calibration and validation testbed. As it played out, UMD developed algorithms utilizing INRIX-provided data, and NREL did the same with TomTom-provided data.

The research results developed by UMD and NREL were cross-checked with each other and reviewed by the project steering committee and by TTI researcher Shawn Turner. This provided a layer of independence and validation that would have otherwise been compromised due to the development roles taken on by UMD and NREL. Note that in the research process both UMD and NREL utilized a method in which a portion of the calibration data was set aside for use in validation and the remaining data was used to create the volume estimator. Then the volume estimator was used on the data that was set aside to measure the fidelity of the volume estimate. 


\section{Task 3-2 Testbed Preplanning}

NREL developed the volume estimation algorithm using data obtained from the Denver metropolitan area. The dataset used for estimation was merged from three data sources TomTom probe vehicle data, CDOT CCS data, and weather information on Weather Underground. TomTom probe vehicle data includes travel time, speed, and probe vehicle count through their GPS devices, as well as road characteristics such as speed limit and functional road class information on every segment of TomTom road network. The TomTom road network covers almost all roads in the United States with an average segment length of 398 feet. CDOT CCS data includes ground truth traffic volume at locations where CCSs have been deployed on Colorado highway system. Weather Underground provides daily weather information such as temperature, visibility, wind, and precipitation.

\section{Task 3-3 Initialize Testbed}

All the data was aggregated on an hourly basis. Three months of data from Feb 1, 2017 to April 30, 2017 for all 14 CCS locations in the Denver region were retrieved and integrated by location and time for modeling. Figure 2 shows the geospatial distribution of CCSs on the Denver roadway network and their matched TomTom segments marked in red.

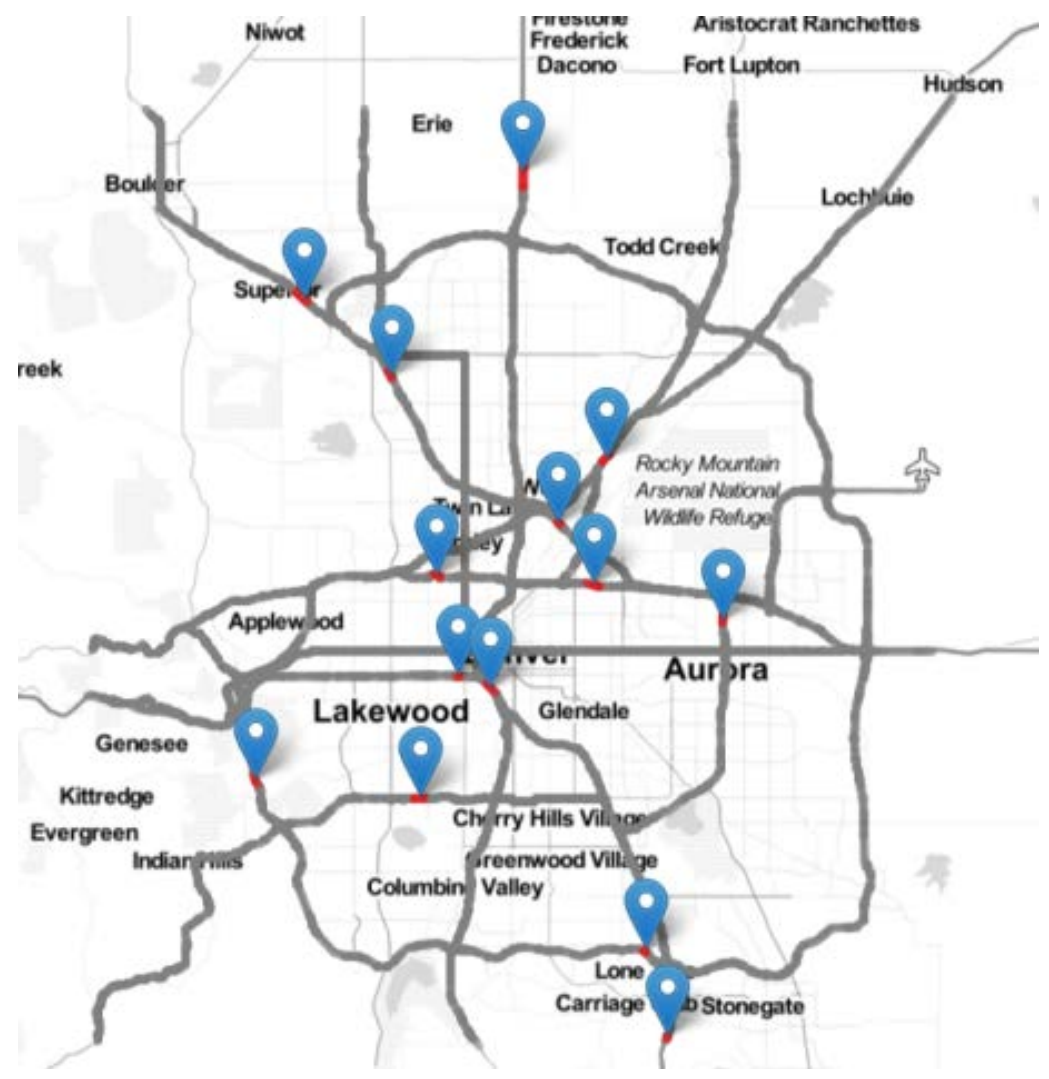

Figure 3 - Geospatial distributions of CCSs on Denver roadway network

The dataset contains 52,092 records (i.e., hours of data), with each CCS ranging from 2700 to 4000 hourly records. The average penetration rate of probe vehicles (i.e., the ratio of number of probe vehicles to the total traffic on a road segment) for each CCS location ranged from $7 \%$ to $12 \%$. The average hourly observed traffic volume at each CCS varies from 1500 to $5000 \mathrm{veh} / \mathrm{hr}$. 


\section{Phase I Deliverables}

The work statement did not include discrete deliverables for Phase 1. However, a 'go/no-go' decision was built into the original project timeline. The 'go/no-go' decision was inserted to provide a sanity check on the initial analysis to make sure there was sufficient evidence to justify continued investment in the research effort. The 'go/no-go' also provided a check point to make sure that all appropriate agreements were in place and partners were on board to support the continuation of the project.

The project reached this decision point in mid-2017. The first technical analysis revealed error to capacity ratios of approximately $10 \%$, which was in agreement with needs revealed by the Coalition survey. Also, the partnerships with probe data vendors, access to calibration data through TMAS and validation data from states were mature and on track for a successful research effort. As such, the project proceeded to Phase 2 activities, including further refinement of algorithms.

\section{PHASE II}

\section{Task 4 Product Development and Refinement}

\section{Task 4-1 Testbed Production}

Given the revised roles of UMD and NREL as algorithm developers, the nature of this task evolved to focus on iterative attempts to develop effective volume estimation techniques, combining industry probe data (both speed/travel time estimates along with number of observed probes) with other data sources such as geometry (number of lanes), weather (temperature and precipitation), and temporal data (time of day, day or week/year).

\section{Task 4-2 Historical Archive Approach}

The model structure is shown in Figure 4. The attribute being modeled (the dependent variable) here is the hourly traffic volume on a segment. This information is available on road segments with CCSs. The input variables (or the independent variables) being used to model the dependent variable are: i) the average hourly speed and probe vehicle count of the past 6 hours on the segment, ii) segment attributes (functional road class, speed limit, lane count, roadway capacity), iii) weather information (temperature and visibility), and iv) temporal information (month, day of week, hour of day).

Speed, probe vehicle counts, speed limit, and functional road class information was provided by TomTom and weather data was obtained from Weather Underground. The independent variables used in the model development process were available for all road segments in the network (i.e., stations with CCSs and stations without CCSs). The CDOT CCS traffic volume data served as the dependent variable for model estimation. 


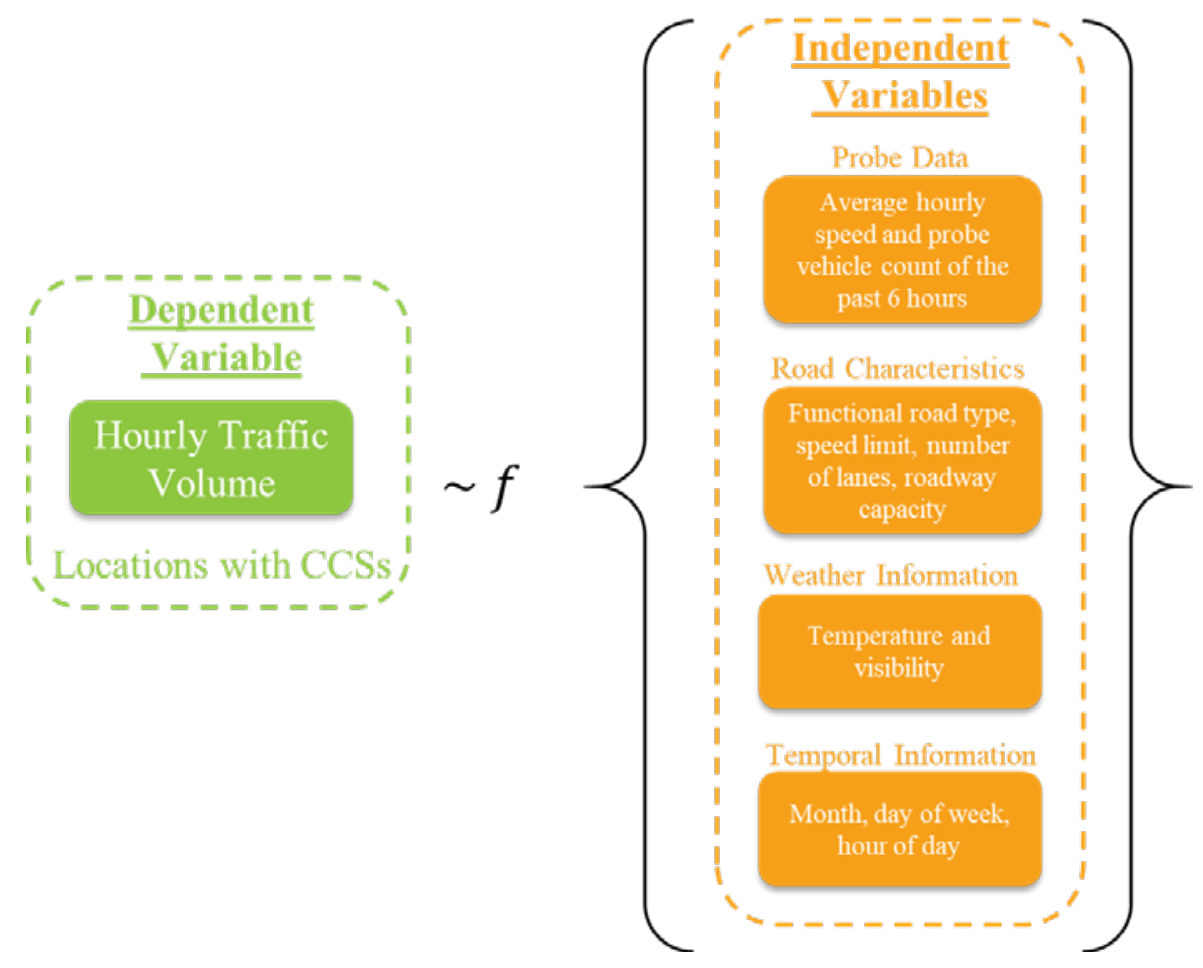

Figure 4 Model structure

The models were developed by estimating the temporal variation of volume for a whole week. The NREL research and development with TomTom data in Colorado was able to achieve results with approximately 5\% error with respect to capacity on freeways. Results from US-36 are illustrated in Figure 3, showing the strong correlation with predictions and observations over a seven day period.

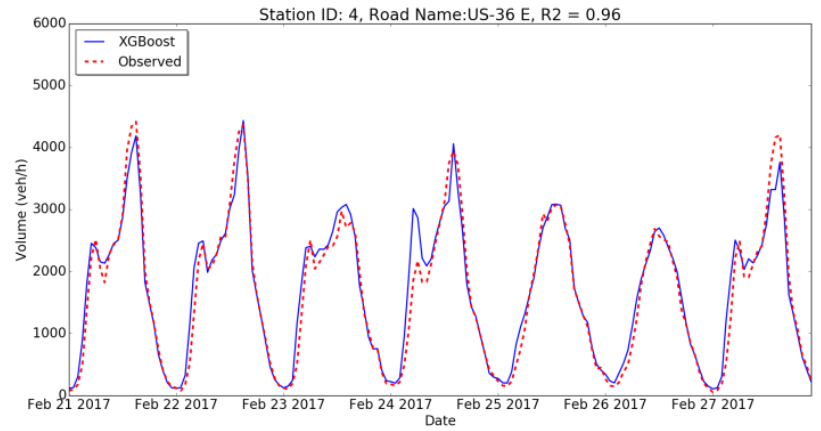

Figure 5 - One-week temporal variation of estimated and actual traffic volume using the XGBoost prediction algorithm

Feedback from the steering committee and other outside reviewers indicated the desire to test the process on non-freeways. As a result, NREL extended the process, using 48-hour counts for calibration (rather than CDOT continuous count stations) as 48-hour counts were prevalent on off-freeway roadways. The off-freeway results revealed results that were stable and within the accuracy target ranges. Representative results for off-freeway results for Colorado's Denver metropolitan region are shown in Table 1, comparing the XGBoost methodology (the NREL machine learning method used with TomTom data) with other methods used to create volume estimates whenever volume data is absent. 
Table 1 - Comparison of NREL Machine Learning volume estimator with prevailing methods for non-freeways.

\begin{tabular}{|l|c|c|}
\multicolumn{1}{c}{ Model } & $\begin{array}{c}\text { MAPE } \\
\text { (Vol>20) }\end{array}$ & $\begin{array}{c}\text { EMFR } \\
\text { (Vol>20) }\end{array}$ \\
\hline XGBoost & $29.7 \%$ & $10.8 \%$ \\
\hline Linear Regression & $90.4 \%$ & $20.5 \%$ \\
\hline AADT Based Method & $124.9 \%$ & $28.1 \%$ \\
\hline
\end{tabular}

After the models were developed and validated, they were put to test to estimate traffic volume on all freeways and major highways (i.e., locations with and without CCSs) in the Denver metropolitan region. Figure 6 depicts these results and serves as illustration for how we can harness the power of machine learning algorithms to develop volume estimation models that can predict traffic volume on all freeways and major highways in the Denver metro region.

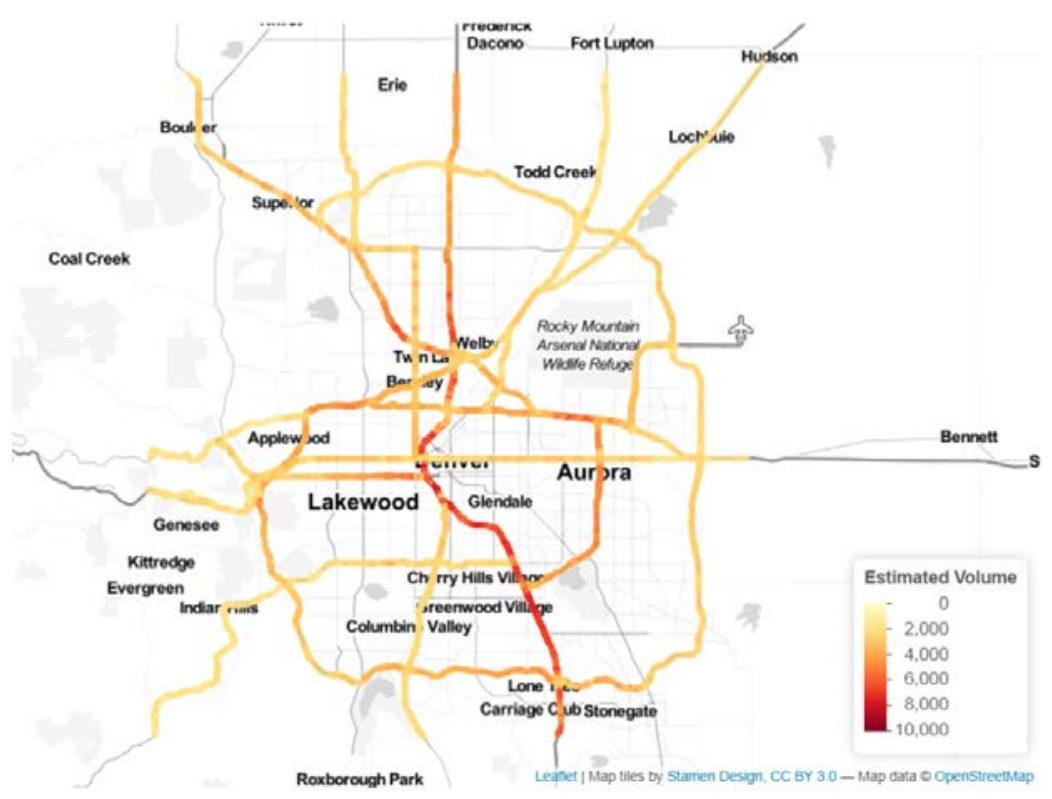

Figure 6 - Traffic volume estimates for the Denver network

\section{Task 4-3 Real-Time Approach}

The data available to the researchers was inherently historical, but the volume estimation methodologies were developed to resemble the data that would be typically available in a realtime environment. Additional work is needed to understand the time-latency and availability of data in a real-time environment. However, the results from the NREL analysis did not indicate any significant degradation in fidelity, though it should be noted that real-time processes of any sort are highly subject to communications reliability and latency, which could not be tested and replicated in a research environment.

A key consideration in real-time traffic volume estimation is computation time. The computation time of estimating one hour's volume (shown in Figure 6) for the three models was recorded and the findings are presented in Table 2. Each approach provides volume estimates within a half second. The low computational time of XGBoost in both training and estimation allows makes it ideal for real time applications. 
Table 2 Computation Time

\begin{tabular}{|l|l|l|}
\hline Model & Training & Estimation \\
\hline RF & $73 \mathrm{~s}$ & $0.37 \mathrm{~s}$ \\
\hline GBM & $124 \mathrm{~s}$ & $0.27 \mathrm{~s}$ \\
\hline XGBoost & $13 \mathrm{~s}$ & $0.13 \mathrm{~s}$ \\
\hline
\end{tabular}

\section{Phase II Deliverables}

The results presented above were presented to the coalition and presented in academic venues. The following paper, which provides in-depth detail, is currently in review for publication in an academic/industry journal, but may be published by NREL if not published in such journals:

- Hou, Y., Young, S.E., Dimri, A., Garikapati, V., and N. Cohn, "Network Scale Real-Time Ubiquitous Volume Estimation Using Tree-Based Ensemble Learning Methods", Submitted to Expert Systems with Applications.

\section{Modification 1: No Cost Time Extension}

The project duration was extended by 12 months with no change to the work statement.

\section{Modification 2: Machine Learning Techniques}

Task 1: Under Modification 2, NREL explored advanced machine learning algorithms to estimate traffic volume. Work completed under Modification 2 is incorporated into the findings presented above. In this section, additional detail regarding the methodology and validation is presented.

Task 2: Modification 2 efforts included characterizing the anticipated accuracy of volume estimates using statistical evaluation methods commonly accepted in the research community. Table 3 presents the overall MAPE, and ECR values for all the methods used for comparing the results of the tree-based ensemble learning methods. After the leave-one-location-out (LOLO) validation process, each observed value in the dataset is mapped to an estimated value of the hourly volume. The overall MAPE and ECR values of tree-based models are $60 \%$, and $30 \%$ lower than other methods tested, respectively. When compared with AADT-based TTI methods, MAPE and ECR values of tree-based models are $50 \%$ and $30 \%$ lower, respectively.

Table 3 - Comparison with Linear Regression

\begin{tabular}{|l|l|l|}
\hline Model & Overall MAPE & Overall ECR \\
\hline RF & $17.8 \%$ & $5.2 \%$ \\
\hline GBM & $18.3 \%$ & $4.8 \%$ \\
\hline XGBoost & $17.7 \%$ & $5.3 \%$ \\
\hline LSTM & $25.9 \%$ & $6.3 \%$ \\
\hline Linear & $41.8 \%$ & $7.2 \%$ \\
\hline TTI Method & $35.8 \%$ & $7.2 \%$ \\
\hline
\end{tabular}


One important feature of tree-based ensemble models is that variable importance can be constructed to understand the mechanism of the modeling process (Breiman, 2001). For GBM and XGBoost, the most relevant variables are the recent hourly probe counts and average speed in the past a few hours, and hour of day. The relative higher importance of probe count and average speed features with larger time difference ( $t-5, \mathrm{t}-4$ etc.,) compared to those features with smaller time difference ( $\mathrm{t}-1, \mathrm{t}-2$ etc.,) is somewhat counterintuitive. However it should be noted that: i) including probe count and average speed from multiple time periods before the current hour might be creating some confounding effects as these variables are closely correlated, and ii) the scale for depicting relative importance of GBM and XGBoost is extremely small meaning that the relative importance of average speed at time $(\mathrm{t}-5)$ is not all that different from the importance of average speed at time (t-1). Efforts are currently underway to improve the estimation accuracy of the model by adding more uncorrelated independent variables (such as network topology, spatial indicators, and socio-demographic variables).

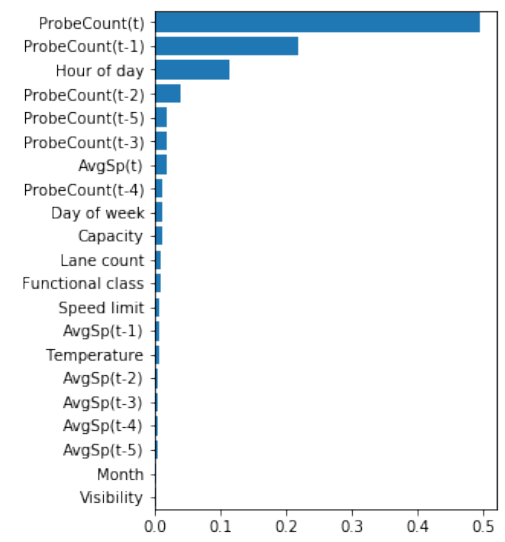

(a)

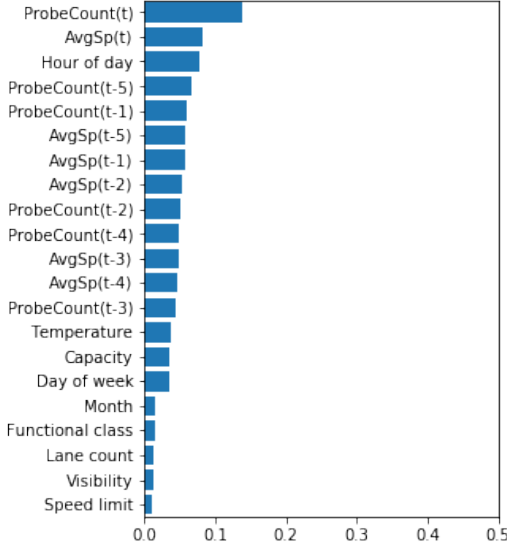

(b)

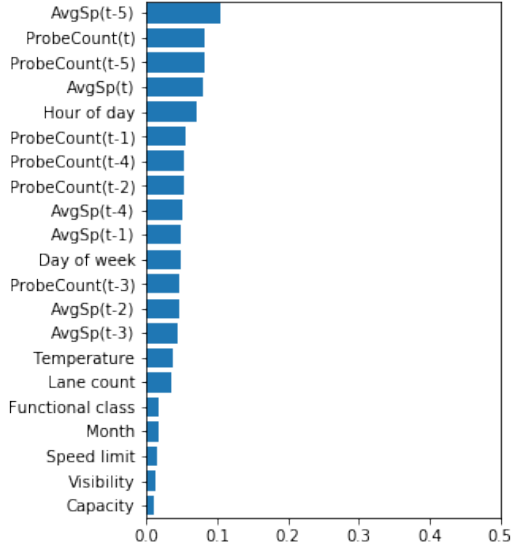

(c)

Figure 7 - Relative importance of input variable (a) RF (b) GBM (c) XGBoost

Task 3: In addition to providing data for accomplishing tasks 1, and 2, TomTom and NREL submitted a proposal to DOE's Technology Commercialization Fund that can enable commercializing the lab developed volume estimation methodology.

\section{Modification 3: Algorithm Testing and Refinement}

Task 1: Under Modification 3, NREL intended to test the spatial transferability of the algorithms developed earlier in the project. However, due to budget and data availability constraints, this task could not be accomplished. This was communicated and conferred with the project sponsor.

Task 2: Under this mod, NREL also evaluated and refined metrics to account for unusual events, and limitations imposed by the training sets. The figure below shows representative results from application of the volume prediction method, where predicted volumes (blue line) and actual traffic volume counts (red dashed line) on a roadway segment are plotted for the duration of a week. The methodology not only provides accurate volume predictions for both peak and offpeak traffic conditions, but also captures the abnormal volume variations during inclement weather (a snowstorm that occurred on Feb. 23, 2018). These findings confirmed the strength of probe data derived volume estimates. 


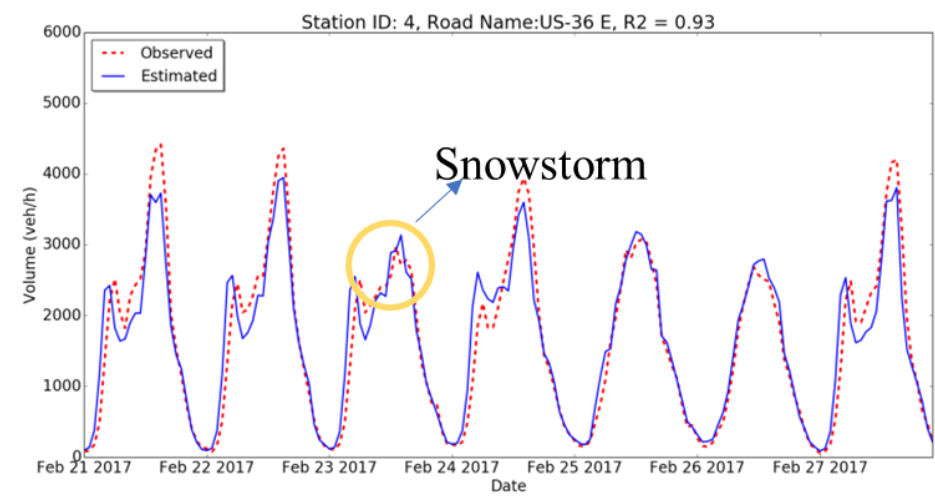

Figure 8 - One-week temporal variation of estimated and actual traffic volume using the XGBoost prediction algorithm

Task 3: The research completed under modification 3 explored the relationship between estimation accuracy and probe penetration rates and whether penetration rates may influence confidence measures. The table below shows the confidence measures (for Colorado freeway volume estimation) computed with and without utilizing probe vehicle data.

Table 4 - Impact of Probe Counts on Estimation Accuracy

\begin{tabular}{|l|l|l|l|}
\hline & MAE & EMFR & \multicolumn{1}{|c|}{$\boldsymbol{R}^{2}$} \\
\hline Without Probe Data & 875 & $12.4 \%$ & 0.65 \\
\hline With Probe Data & 357 & $5.3 \%$ & 0.91 \\
\hline
\end{tabular}

As observed from Figure 7 and Table 4, including probe counts in the volume estimation methodology significantly improves prediction accuracy (i.e., decrease in MAE, and EMFR values) coupled with an increase in the goodness of fit measure $\left(\mathrm{R}^{2}\right)$. So, it can be expected that a higher penetration of probe vehicles on roadways will help increase the estimation accuracy of the model.

This task has led to the submission of a software record claim (SWR 17-20).

\section{$\underline{\text { References }}$}

Sekula, P., N. Markovic, Z. Vander Laan, and K.F. Sadabadi, 2018. "Estimating Historical Hourly Traffic Volumes via Machine Learning and Vehicle Probe Data: A Maryland Case Study," Transportation Research Part C: Emerging Technologies 97 (December): 147-158. https://doi.org/10.1016/j.trc.2018.10.012.

\section{Subject Inventions Listing:}

None

ROI \#:

None 


\section{Responsible Technical Contact at Alliance/NREL:}

Stan Young | $\underline{\text { Stanley.Young@nrel.gov }}$

Name and Email Address of POC at Company:

Denise Markow | dmarkow@i95coalition.org

DOE Program Office:

U.S. Department of Energy Office of Energy Efficiency and Renewable Energy, Vehicle Technologies Office 